\title{
IMPORTANCIA DEL CAMBIO GENERACIONAL PARA EL DESARROLLO DE LA CIENCIA Y LA TECNOLOGÍA EN MÉXICO.
}

\author{
Deneb Elí Magaña Medina*, Norma Aguilar Morales**, Edith Georgina Surdez Pérez ${ }^{* * *}$.
}

\author{
Magaña-Medina D.E., Aguilar-Morales N., Surdez-Pérez \\ E.G.. Importancia del cambio generacioanl para el \\ desarrollo de la ciencia y la tecnoloigía en México. Hitos de \\ Ciencias Económico \\ Administrativas 2012;18 (50):35-40.
}

\section{RESUMEN}

La educación hasta hace medio siglo, giraba en torno a la alfabetización, hoy día es necesario evolucionar hacia una alfabetización científica como objetivo primordial de los sistemas educativos. Sobre este aspecto es importante considerar que el segmento de la población en el país habilitado para la investigación científica y tecnológica está envejeciendo.

Es prioritario considerar este hecho en las políticas de educación superior y desarrollar estrategias para realizar el cambio generacional que permita desarrollar entre los estudiantes el interés y competencias necesarias para la realización de las diversas actividades científicas y tecnológicas que México requiere.

\author{
Magaña-Medina D.E., Aguilar-Morales N., Surdez-Pérez \\ E.G.. Importance of generational change for the \\ development of science and technology in mexico.Hitos \\ de Ciencias Económico \\ Administrativas 2012;18 (50):35-40.
}

\section{ABSTRACT}

Education, until half a century ago, centered its attention on literacy. Nowadays it is necessary to move towards a scientific literacy as the primary objective of the education systems. On this aspect it is important to consider that the segment of the population in the country entitled to the scientific and technological research is aging.

It is a priority to consider this fact in the high education policies, and to develop strategies to make the generational change in a way that allows students to develop interest and skills needed to carry out the various scientific and technological activities that Mexico requires.
Palabras clave: Sociedad del Conocimiento. Cambio Generacional. Ciencia y Tecnología.
Key words: Society of the Knowledge. Generational Change. Science and Technology.

DIRECCIÓN PARA RECIBIR CORRESPONDENCIA: Correo electrónico: deneb_72@yahoo.com

I siglo $\mathrm{XXI}$, se ha caracterizado por un crecimiento de la economía del conocimiento, en la cual, la generación y explotación del conocimiento, juega un rol predominante en la creación de riqueza.

García (2004) afirma que la inserción eficiente de la institución universitaria en la sociedad del conocimiento requiere un cambio en la concepción tradicional para que ésta pueda lograr un desarrollo sostenido.

La Organización para la Cooperación y el Desarrollo Económico (OCDE) plantea que hace 50 años, el objetivo de la educación giraba en torno a la alfabetización, sin embargo, hacia finales del siglo pasado, se remarcó la importancia ya no sólo de

\footnotetext{
* Doctora en Ciencias Administrativas: Gestión Socioeconómica. Profesora-Investigadora. División Académica de Ciencias Económico Administrativas. Universidad Juárez Autónoma de Tabasco (UJAT).

** Maestra en Administración. Profesora-Investigadora. División Académica de Ciencias Económico Administrativas. Universidad Juárez Autónoma de Tabasco (UJAT).

*** Maestra en Administración. Profesora-Investigadora. División Académica de Ciencias Económico Administrativas. Universidad Juárez Autónoma de Tabasco (UJAT).
}

Fecha de recibido: 9 de septiembre de 2011 Fecha de aceptación: 4 de octubre de 2011. 
la alfabetización, sino la imperante necesidad de evolucionar a una alfabetización científica como objetivo primordial de los sistemas educativos.

Hoy día, se reconoce a la sociedad del conocimiento como la base del desarrollo económico, y se remarca la importancia de la enseñanza científica y tecnológica como un instrumento esencial no sólo para la formación de los científicos, sino también para fomentar los conocimientos básicos, las aptitudes prácticas y el espíritu crítico de los niños, los jóvenes y los adultos, (Organización de las Naciones Unidas para la Educación, la Ciencia y la Cultura [UNESCO], 2006, p.1).

\section{Antecedentes.}

La relación entre docencia e investigación desde la formación de las modernas universidades europeas durante el siglo XIX fue conceptuada de dos formas: la primera, comola unidad entre docencia e investigación, que fue la base de la tradición universitaria alemana de Humboldt; mientras que la segunda, fue la separación de ambos procesos, el fundamento de la universidad napoleónica; cuya tradición fue seguida en México y España (Rivas, 2004; Guerrero y González, 2011).

Un referente histórico de la universidad alemana de Humboldt en Berlín, es que atribuyen el éxito del modelo al fomento de las ciencias y las artes mediante la investigación, la enseñanza y el estudio profundo de los temas. Sus principios básicos sostienen que la docencia e investigación son elementos de un mismo proceso; en consecuencia la generación de conocimiento científico implica el ejercicio de la razón para conocer, explicar, proyectar y prever el actuar humano y el funcionamiento de la naturaleza; por lo tanto, la investigación ejercita a la razón (Quintero, Munévar y Munévar, 2008).

\section{El Sistema Nacional de Investigadores.}

Las políticas educativas y de fomento a la ciencia y la tecnología en México, han ido evolucionando y mejorando las estrategias para alcanzar el desarrollo económico a pesar de que México aún no logra superar el $0.5 \%$ del P.I.B. en actividades de ciencia y tecnología.
Sobre este punto, es de interés reflexionar sobre el hecho de que la población en el país habilitada para la investigación científica y tecnológica está envejeciendo, por lo que, para llevar a cabo el cambio generacional en el desarrollo científico y tecnológico, es prioritario fomentar entre los estudiantes de todos los niveles educativos el interés por la ciencia y la tecnología. Es preciso tomar en cuenta que la preparación de nuevos científicos y tecnólogos es de largo plazo y de altos costos (Consejo Nacional de Ciencia y Tecnología [CONACYT], 2010).

Sánchez (2010) señala sobre esta problemática que uno de los actores fundamentales para el impulso de los jóvenes en las actividades científicas y tecnológicas de México, es el Sistema Nacional de Investigadores (SNI) del Consejo Nacional de Ciencia y Tecnología (CONACYT), el cual se ha convertido en una pieza fundamental del Sistema de Educación Superior y Científico del país.

El Sistema Nacional de Investigadores (SNI) fue creado por acuerdo presidencial publicado en el Diario Oficial de la Federación el 26 de julio de 1984, para reconocer la labor de las personas dedicadas a producir conocimiento científico y tecnología. El reconocimiento se otorga a través de la evaluación por pares y consiste en otorgar el nombramiento de investigador nacional. EI SNI tiene por objeto promover y fortalecer, a través de la evaluación, la calidad de la investigación científica y tecnológica, y la innovación que se produce en el país. El Sistema contribuye a la formación y consolidación de investigadores con conocimientos científicos y tecnológicos del más alto nivel como un elemento fundamental para incrementar la cultura, productividad, competitividad y el bienestar social (CONACYT, 2010).

Tomando como referente al SNI, se puede apreciar en la Figura 1, tomada del Atlas de la Ciencia Mexicana [ACM] (2010) el incremento en la edad promedio de sus miembros, que pasa de 40 años en 1984 a 49 años de edad en el 2009 (Figura 1).

En un análisis por rangos de edad con relación al nivel (Tabla 1) se puede apreciar que solo el $23.28 \%$ de los miembros del SNI, son menores a 39 años de edad y tan solo el .22\% menor a 30 años. En contraposición el $41.59 \%$ de sus miembros son mayores a 50 años. 


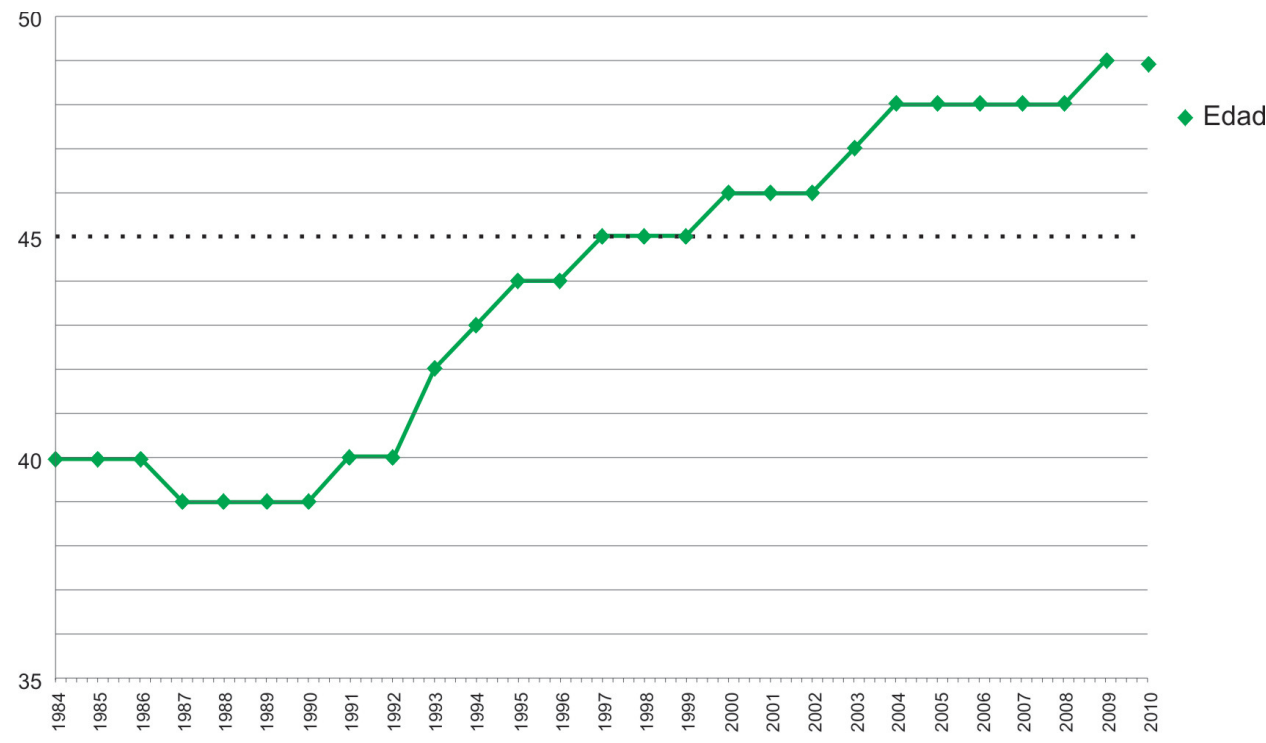

Fuente: Atlas de la Ciencia Mexicana (2010).

FIGURA 1. EVOLUCIÓN DE LA EDAD PROMEDIO DE LOS INVESTIGADORES EN EL SISTEMA NACIONAL DE INVESTIGADORES 1984-2010.

El Acervo de Recursos Humanos en Ciencia y Tecnología.

En el Manual de Canberra se defineal Acervode Recursos Humanos en Ciencia y Tecnología (ARHCyT) como el subconjunto de la población que ha cubierto satisfactoriamente la educación de tercer nivel de acuerdo con la Clasificación Internacional Normalizada de la Educación (ISCED), en un campo de la ciencia y la tecnología; y/o está empleada en una ocupación de ciencia y tecnología que generalmente requiere estudios de tercer nivel.

El tercer nivel de acuerdo

Las cifras soportan la inquietud de evaluar la problemática hacia el interior de las Instituciones de Educación Superior, con la finalidad de que se generen las estrategias que permitan la evolución de la investigación en México y para ello es relevante que se considere la habilitación del recurso humano calificado para el desarrollo de la ciencia y la tecnología. con la ISCED comprende los niveles educativos posteriores al bachillerato, estudios conducentes a grados universitarios o superiores (ISCED 5A: licenciaturas; ISCED 6: especialidades maestrías y doctorados) y estudios no equivalentes a los universitarios pero que crean habilidades específicas (ISCED 5B: carreras de técnico superior universitario). Las ocupaciones consideradas como de ciencia y tecnología son un subconjunto de

TABLA I. DISTRIBUCIÓN POR RANGO EDAD Y NIVEL DE LOS INVESTIGADORES EN EL SNI - 2008.

\begin{tabular}{lccccrc}
\hline \multicolumn{1}{c}{ Rango de edad } & C & I & II & III & TOTAL & $\%$ \\
\hline Menores de 30 años & 24 & 7 & 1 & 0 & 32 & $0.22 \%$ \\
de 30 a 39 años & 1775 & 1519 & 61 & 2 & 3357 & $23.06 \%$ \\
de 40 a 49 años & 773 & 3430 & 811 & 102 & 5116 & $35.14 \%$ \\
De 50 a 59 años & 9 & 2464 & 1262 & 395 & 4130 & $28.37 \%$ \\
de 60 a 60 años & 0 & 596 & 489 & 366 & 1451 & $9.97 \%$ \\
más de 70 años & 0 & 104 & 153 & 216 & 473 & $3.25 \%$ \\
\hline Total & 2581 & $\mathbf{8 1 2 0}$ & 2777 & $\mathbf{1 0 8 1}$ & $\mathbf{1 4 5 5 9}$ & $\mathbf{1 0 0 \%}$ \\
\hline
\end{tabular}

Fuente: Atlas de la Ciencia Mexicana (2010). 
las ocupaciones consideradas en la Clasificación Internacional Normalizada de Ocupaciones, ISCO' (CONACYT, 2010, p. 32).

La información referente al ARHCyT en los países de la OCDE señala que $24.3 \%$ en promedio de la población ocupada cuenta con estudios de tercer nivel, con un amplio margen de variación, desde $11.7 \%$ en Turquía, hasta $35.7 \%$ en Bélgica. Por su parte, Finlandia (34.9\%) y España (31.2\%) se encontraban por encima del promedio de la Unión Europea (23.2\%), mientras México ocupa los últimos lugares con $20.9 \%$ de la población ocupada con estudios de licenciatura, sólo por arriba de Italia y Portugal. En términos de población ocupada, México está en desventaja en relación con la mayoría de las naciones de la OCDE, ya que la fuerza laboral está conformada en su mayoría por personas poco calificadas, a diferencia de otros países que tienen una mano de obra con un nivel académico superior (CONACYT, 2010, p. 34).

En la Tabla II se puede apreciar que el ARHCyT es superior a cinco millones de personas, de las cuales $56 \%$ desempeña alguna actividad científica o tecnológica, mientras que el $44 \%$ restante está dedicado a otras funciones. Así, dos millones y medio de personas podrían incorporarse a labores vinculadas con el conocimiento científico y tecnológico, pero por diversas circunstancias efectúan otro tipo de tareas (CONACYT, 2010, p.37).

\section{Reflexiones sobre la Problemática.}

Las cifras planteadas, revelan que se cuenta con el potencial necesario para el desarrollo de actividades científicas y tecnológicas que permitan a México, hacer competitiva su economía basándola en el conocimiento.

TABLA II. ACERVO DE RECURSOS HUMANOS EN CIENCIA Y TECNOLOGÍA (ARHCYT) 2006 - 2009. POBLACIÓN QUE COMPLETÓ EXITOSAMENTE EL NIVEL DE EDUCACIÓN ISCED 5 Ó SUPERIOR Y/O ESTÁ OCUPADA EN ACTIVIDADES DE CIENCIA Y TECNOLOGÍA.

\begin{tabular}{|c|c|c|c|c|c|c|c|c|c|}
\hline & & \multicolumn{4}{|c|}{ Miles de Personas } & \multicolumn{4}{|c|}{$\begin{array}{l}\text { Participación con respecto al total de la } \\
\text { población de } 18 \text { años ó más }\end{array}$} \\
\hline & & 2006 & $2007^{*}$ & $2008^{*}$ & $2009^{*}$ & 2006 & $2007^{\star}$ & $2008^{*}$ & $2009^{*}$ \\
\hline TOTAL & & $8,688.50$ & $9,263.60$ & $9,540.20$ & $9,816.90$ & 13.23 & 13.65 & 13.83 & 14.01 \\
\hline \multicolumn{10}{|l|}{ Género } \\
\hline & Hombres & $4,657.90$ & $4,816.50$ & $4,932.30$ & $5,048.00$ & 7.10 & 7.07 & 7.11 & 7.14 \\
\hline & Mujeres & $4,030.70$ & $4,447.00$ & $4,608.00$ & & 6.14 & 6.59 & 6.73 & 6.87 \\
\hline \multicolumn{10}{|c|}{ Ocupación } \\
\hline & Directivos & 880.40 & 839.80 & 849.00 & 892.70 & 1.34 & 1.24 & 1.23 & 1.22 \\
\hline & Profesionales & $3,026.20$ & $3,146.70$ & $3,240.90$ & $3,373.30$ & 4.61 & 4.71 & 4.78 & 4.85 \\
\hline & Técnicos & $1,481.70$ & $1,371.50$ & $1,402.10$ & $1,470.80$ & 2.26 & 2.04 & 2.05 & 2.06 \\
\hline & $\begin{array}{l}\text { Otras } \\
\text { ocupaciones }\end{array}$ & $2,157.00$ & $2,462.10$ & $2,553.70$ & $2,608.90$ & 3.29 & 3.61 & 3.69 & 3.77 \\
\hline & Desocupados & 183.00 & 150.60 & 152.50 & 118.10 & 0.28 & 0.18 & 0.17 & 0.16 \\
\hline & Inactivos & 960.20 & $1,293.00$ & $1,341.30$ & $1,353.10$ & 1.46 & 1.87 & 1.91 & 1.95 \\
\hline \multicolumn{10}{|c|}{ Educación } \\
\hline & Posgrado & 512.00 & 529.40 & 552.10 & 574.80 & 0.78 & 0.77 & 0.79 & 0.82 \\
\hline & Licenciatura & $5,632.50$ & $5,875.40$ & $6,079.20$ & $6,282.90$ & 8.58 & 8.77 & 8.95 & 9.12 \\
\hline & Técnica & 428.00 & 883.00 & 902.10 & 921.30 & 0.65 & 1.29 & 1.29 & 1.29 \\
\hline & $\begin{array}{l}\text { Gradosmenores } \\
\text { al técnico }\end{array}$ & $2,101.40$ & $1,909.60$ & $1,935.90$ & $1,962.10$ & 3.20 & 2.78 & 2.75 & 2.73 \\
\hline & Sin instrucción & 14.60 & 36.30 & 38.40 & 40.50 & 0.02 & 0.03 & 0.03 & 0.03 \\
\hline & No Especificado & 0.10 & 29.90 & 32.60 & 35.20 & 0.00 & 0.02 & 0.02 & 0.02 \\
\hline
\end{tabular}

${ }^{*}$ Cifras Estimadas. A partir de 2006 se refiere a la población catalogada como disponible de acuerdo con la definición de la Encuesta Nacional de Ocupación y Empleo.

Fuente: INEGI-STPS, Bases de datos de la Encuesta Nacional de Empleo, varios años. INEGI, Base de datos de la muestra censal, varios años.CONACYT, 2010, p. 229.

${ }^{1}$ Fuentes: OCDE, Manual on the measurement of human resources devoted to S\&T «Canberra Manual», 1995. UNESCO, International Standard Classification of Education, ISCED, 1997. ILO, International Labor Office, International Standard Classification of Occupations, ISCO, 1988(citado por CONACYT, 2010, p. 32). 
Sin embargo, es imperativo considerar que realizar investigación científica es muy caro, y sin duda, es una de las actividades más costosas para las instituciones educativas, sobre todo si se considera que sus resultados suelen presentarse a largo plazo y esto la convierte en una actividad poco atractiva, siendo una de las muchas causas por las cuales los jóvenes científicos mexicanos, lejos de recibir apoyos y estímulos para desarrollarse, son lanzados al desempleo o al subempleo en las grandes ciudades (Amador y López, 2011).

Didou y Gérard (2011) afirman que la plantilla académica en México da escasa cabida a los jóvenes doctores que México se precia de formar y esto se debe en gran parte a que los académicos de mayor edad no contemplan en el corto plazo la jubilación.

Los sistemas de retiro para los académicos en México, según el estudio de Bensunsán y Ahumada (2006), no ofrecen un ingreso que permita sostener el nivel de vida similar al que se tiene antes del retiro. La brecha en los ingresos y la pérdida de prestaciones contractuales constituyen el principal factor a la hora de postergar el ejercicio de este derecho.

Desde una perspectiva financiera, lo costoso de la inversión en un activo intangible como lo es el capital humano, hace difícil la tarea de los gestores de recursos económicos para las organizaciones educativas y por ende la evaluación y redireccionamiento de los programas debe enfocarse a indicadores que permitan su evaluación continua y la mejora de los mismos.

Esta situación demanda el diseño y adopción de diversas estrategias dirigidas al fomento de las capacidades de investigación con miras a generar el acervo humano que requiere México para el desarrollo de las actividades científicas y tecnológicas, pero contemplando el espacio que ha de ocupar en los diversos sectores económicos.

Sobre esta problemática, se concluye que las instituciones deben actuar en tres vertientes principales. La primera debe centrar la estrategia en la generación de programas orientados a comenzar una verdadera vocación de los jóvenes hacia las actividades científicas y tecnológicas que permitan contar con el factor humano requerido. La segunda vertiente debe considerar los sistemas de jubilación y retiro que permita condiciones dignas de jubilación para dar cabida a los jóvenes egresados en los espacios académicos. Por último se considera que la mirada del sector productivo hacia la ciencia, debe dejar el rol pasivo que lo ha caracterizado para seguir evolucionando con base en el conocimiento que genera, y como principal fuente de captación de recursos humanos de alto nivel, que le permitan ser competitivo en la economía del conocimiento.

Para la primera vertiente, Hernández (2005) propone a los Semilleros de Investigación como una propuesta esencialmente estudiantil hacia el interior de las universidades, que busca la formación en investigación en el marco de una cultura académica con valores y principios distintos a los tradicionales, los cuales hacen posible al estudiante visualizar una forma diferente de entender y asumir el espíritu científico.

Con respecto a los sistemas de retiro en las organizaciones educativas, Bensunsán y Ahumada (2006) señalan que se hace necesaria la adopción de un sistema de retiro complementario de cotización definida, alternativa que ha generado una gran discusión dentro de algunas instituciones.

Por otra parte, las universidades y centros de investigación deberían centrar sus esfuerzos en institucionalizar o, en su caso, renovar sus programas de carrera académica. En forma complementaria, el diseño de políticas institucionales que permitan una inserción adecuada de los jóvenes académicos y mejoren los espacios de interacción entre los profesores-investigadores de distintas generaciones $y$, muy en particular, que creen la oportunidad para que los eméritos o académicos distinguidos compartan sus logros y experiencias con los que se inician en la carrera académica (Bensunsán y Ahumada, 2006).

La tercera vertiente, centra su atención en el sector productivo como eje para el desarrollo de actividades científicas y tecnológicas, reforzando la política nacional de apoyo. Sobre esta posición, las organizaciones educativas, deben vincular sus programas de formación hacia el sector productivo y diversas entidades del sector público que pueden dar cabida a esta nueva generación de doctores capacitados para realizar investigación y desarrollo tecnológico. 
Sea cual fuere la estrategia, es imprescindible que la institución universitaria en esta nueva visión de inclusión hacia las actividades de investigación y desarrollo científico, tomen en cuenta el relevo generacional entre las políticas que rigen sus actividades académicas y científicas, porque de otra forma, cualquier programa que no contemple este factor está destinado a perder su trascendencia al paso del tiempo.

\section{REFERENCIAS}

Amador, E. y López, L.J. (2011). Incorporación temprana a la investigación. Cuadernos de Educación y Desarrollo, 3(28). 1-9. Recuperado de http://www. eumed.net/rev/ced/28/amlr.htm

Atlas de la Ciencia Mexicana [ACM] (2010). Atlas de la Ciencia Mexicana 2010. México, D.F. Red temática del CONACyT Complejidad, Ciencia y Sociedad. Recuperado de http://www.atlasdelacienciamexicana. org/es/sni.html

Bensunsán, G., y Ahumada, Í. (2006). Sistemas de jubilación en las instituciones públicas de educación superior y composición por edad del personal académico. Revista de la Educación Superior, XXXV (138), 7-35.

Consejo Nacional de Ciencia y Tecnología [CONACYT], (2010). Informe General del Estado de la Ciencia y la Tecnología. México Autor.

Didou, A., y Gérard, E. (2011). El Sistema Nacional de Investigadores en 2009. ¿Un vector para la internacionalización de las élites científicas? Perfiles Educativos, 33(132), 29-47. Recuperado de http://www.redalyc.org/src/inicio/ArtPdfRed. jsp?iCve=13218510003

García, L.N. (2004). Estrategias de gestión para la capitalización del conocimiento en el contexto de la relación universidad. Sector productivo. Educere. 8(27). 507-516. Recuperado de http://redalyc.uaemex. $\mathrm{mx} /$ redalyc/src/inicio/ArtPdfRed.jsp?iCve=35602709

Guerrero, T. y González, F. (2011). La generación de conocimiento científico en relación con sus efectos en la sociedad: análisis comparativo de la situación en España y México. Tesis para obtener el grado de doctor en educación de la Facultad de Educación de la Universidad Complutense de Madrid. Recuperada http://eprints.ucm.es/13189/1/T33136.pdf

Hernández, U. (2005) Propuesta Curricular para la consolidación de los Semilleros de Investigación como espacios de Formación Temprana en Investigación. Revista Electrónica de la Red de Investigación Educativa [en línea], 1(2). 1-13. Recuperado de http:// revista.iered.org/v1 $2 / \mathrm{html} /$ uhernandez.html

Organización de las Naciones Unidas para la Educación, la Ciencia y la Cultura [UNESCO] (2006, 16-22 de octubre). Enseñanza de las ciencias y la tecnología. Texto de Información en el contexto del $60^{\circ}$ aniversario de la UNESCO. Oficina de Información Pública Memobpi, sitio web UNESCO. Recuperado de http://www.unesco.org/bpi/pdf/memobpi59_ scienceeduc_es.pdf

Quintero, J., Munévar, R., y Munévar, F. (2008). Semilleros de investigación: una estrategia para la formación de investigadores. Educación y Educadores, 11(001), 31-42. Recuperado de http:// pensamientoycultura.unisabana.edu.co/index.php/ eye/article/viewArticle/716/1694.

Rivas, L.A. (2004). La formación de investigadores en México. Perfiles Latinoamericanos, 025. 83113.Recuperado de http://redalyc.uaemex.mx/ pdf/115/11502504.pdf.

Sánchez, H. (2010). 25 años del Sistema Nacional de Investigadores (SNI) y su impacto en las Políticas de Evaluación de la Educación Superior en México. Matices, 5(13), 219-236. 